Stefan Oschatz*, Thomas Reske, Jana Markhoff, Thomas Eickner, Klaus-Peter Schmitz, Niels Grabow and Sabine Illner

\title{
Degradable dual co-electrospun polyester based nonwovens for guided tissue regeneration
}

\begin{abstract}
In this study, initial experiments are presented regarding the suitability of polyester based nonwoven composite materials for guided tissue regeneration based on degradation. The in vitro degradation of poly(lactic-coglycolic acid)/polydioxanone and polycaprolactone/ polydioxanone mats has been performed under accelerated condition in alkaline glycine buffer at $50{ }^{\circ} \mathrm{C}$ for 21 days. The degradation of the materials has been characterized regarding total mass loss and macroscopic morphological changes, as well as determination of the molecular weight degradation via GPC experiments.
\end{abstract}

Keywords: dual co-electrospinning, polyester nonwoven, degradation, guided tissue regeneration

https://doi.org/10.1515/cdbme-2020-3043

\section{Introduction}

Polymer based nonwoven materials are promising candidates for guided tissue regeneration (GTR), especially for the treatment for periodontal damages, as they fulfill several functions. On the one hand, they serve as a barrier for fast growing cells (e.g. gingival cells versus bone cells) into the site of action, as those cells would compete with the target cells.[1] On the other hand, nonwovens may provide a

Corresponding author: Stefan Oschatz, Institute for Biomedical Engineering, Rostock University Medical Center, 18119 RostockWarnemünde, Germany

E-Mail: stefan.oschatz@uni-rostock.de

Thomas Reske and Klaus-Peter Schmitz, Institute for Implant Technology and Biomaterials e.V., 18119 Rostock-Warnemünde, Germany

Sabine IIIner, Jana Markhoff, Thomas Eickner and Niels

Grabow, Institute for Biomedical Engineering, Rostock University Medical Center, 18119 Rostock-Warnemünde, Germany necessary supporting structure for cells and apatite formation to regenerate bone defects.

Electrospinning, in particular, is a versatile tool for the generation of such biomaterials and allows the usage of a vast variety of substrates. Polyesters are especially promising candidates due to their biodegradation ability.

Several approaches for the application of biodegradable nonwovens for GTR have been reported in the literature. As an example, YIN et al. reported on the manufacturing of electrospun poly(lactic acid)/silk fibroin-gelatin scaffold for GTR.[2] These scaffolds showed good cell adhesion for 3T3 mouse fibroblasts and the formation of a cell monolayer in 12 days. In 2016, the group of YAO reported the successful fabrication of a nonwoven from a PDLLA/PLGA blend with an adjustable degradation rate depending on the amount of incorporated PLGA.[3] A different approach was made by ZHANG et al. using an aqueous solution of gelatin to create a degradable nonwoven.[4] MAGIERA et al. went a step further and performed concurrent-electrospinning of PLA with gelatin to create a composite nonwoven structure. The use of this technique resulted in reduced hydrophobicity of the material compared to pure PLA, leading to a faster degradation rate.[5]

In 2019, our group reported on the successful generation of a biostable co-electrospun nonwoven from polyurethane and polyamide.[6] The materials accessible in such a way exhibited distinct fibers of the respective polymer. The approach of this work is the combination of polymers with distinctly different degradation times in a dual co-electrospun composite nonwoven. Such a material may offer a sufficient barrier effect, but, due to the faster degradation of one type of fiber, may still allow for the time delayed migration and colonization of cells. The residual slow degrading polymer acts as supporting scaffold and the opened pores between the fibers enables cell infiltration.

In this work, the degradation behavior of nonwovens consisting of two different polyester fibers is presented. Polydioxanone (PDO) has been combined with poly(lacticco-glycolic acid) (PLGA) and polycaprolactone (PCL). PDO 
(Resomer $\mathrm{X}$ ) is a relatively fast degrading polymer, with degradation times of $<6$ months, according to manufacturer's information. Compared with this, PLGA (Resomer LG 857) exhibits a degradation time of $1-2$ years and PCL $>2$ years.[7] The degradation has been observed in respect to total mass loss and molecular mass degradation.

\section{Materials and Methods}

\subsection{General information:}

Resomer LG 857 S (PLGA) and Resomer X 206 S (polydioxanone) were provided by Evonik. Poly-caprolactone (PCL) was provided by Aldrich.

\subsubsection{Dual co-electrospinning:}

Dual co-Electrospinning was performed on a Contipro 4SPIN C4S LAB2 (Dolní Dobrouč, Czech Republic). The setup used was $24 \mathrm{~cm}$ collector distance, $43 \mathrm{kV}$ high voltage and a feed rate of $50 \mu \mathrm{L} / \mathrm{min}$ for both polymer solutions. All polymers were dissolved in $\mathrm{CHCl}_{3}$ (PDO: $10 \mathrm{w} / \mathrm{w} \%$, PLGA: $6 \mathrm{w} / \mathrm{w} \%$, PCL $7 \mathrm{w} / \mathrm{w} \%)$ prior to spinning. The obtained nonwovens were thermally annealed for $24 \mathrm{hrs}$ at $80{ }^{\circ} \mathrm{C}$ after the spinning process.

\subsubsection{Accelerated degradation:}

Circular samples of $11 \mathrm{~mm}$ diameter were taken from the generated nonwoven and placed in degradation medium without further treatment under decent shaking. For degradation, glycine buffer adjusted to $\mathrm{pH}=8.7$ with $\mathrm{NaOH}$ was used at $50{ }^{\circ} \mathrm{C}$. Medium was replaced after 12 days of degradation. At the selected time points, the degradation medium was removed and the samples were washed twice for 15 min with distilled water. Finally, the samples were dried at $40^{\circ} \mathrm{C}$ under reduced pressure. Mass loss during degradation was investigated by using a Radwag micro balance MYA 0.8/3.4Y.

\subsubsection{GPC-measurements:}

The molecular weight of the polymer nonwoven samples was determined using a PSS SECcurity SEC system (Polymer Standard Services GmbH, Mainz, Germany) including a RI detector combined with a WGE Dr. Bures g 2010 viscosity detector (WGE Dr. Bures GmbH, Dallgow, Germany) equipped with three PSS SDV columns (103, 105 and $106 \AA$, respectively). Chloroform stabilized with ethanol was chosen as mobile phase at a flow rate of $1 \mathrm{~mL} / \mathrm{min}$. The samples were dissolved in chloroform giving a final concentration of $1.5 \mathrm{mg} / \mathrm{mL}$, with $0.2 \mu \mathrm{L} / \mathrm{mL}$ hexylbenzene as internal standard. MW was calculated using twelve polystyrene calibration standards (376 - 2,570,000 g/mol). Column temperature was set to $30{ }^{\circ} \mathrm{C}$.

\section{Results and Discussion}

The degradation behaviour in respect to mass loss can be found in Figure 1. Notably, a distinct difference between the two polymer combinations investigated in this work can be observed. Whereas the PCL/PDO system temporarily stabilizes at around $12 \%$ mass loss after 14 days under the described degradation conditions, the investigated PLGA/PDO composite nonwoven showed a considerably higher mass loss of $\sim 80 \%$ in the same time range.

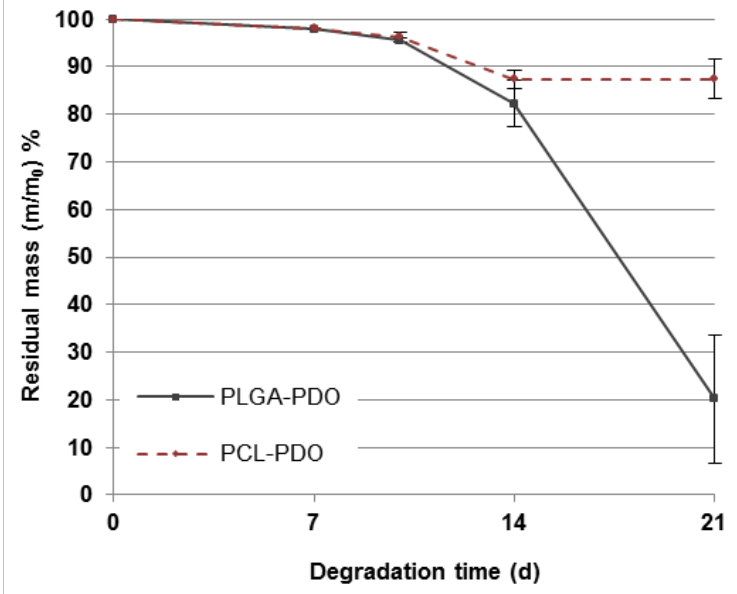

Figure 1: Mass loss during accelerated degradation of PLGA/PDO (grey) and PCL/PDO (red/dashed) composite nonwovens in glycine buffer at $\mathrm{pH}=8.7$ and $50{ }^{\circ} \mathrm{C}(\mathrm{n}=3)$

However, the observed mass loss of PLGA/PDO composite nonwoven was significantly higher than expected based on data from pure PDO and PLGA film material. This can be explained on the different surface-area-to-volume ratio of bulk materials compared to fibrous structures. Since degradation of these materials is based on hydrolysis of the ester groups, a larger surface promotes the diffusion of water soluble molecular fragments at late stages of degradation [8] and may decrease mechanical resilience. In accordance to this, the PLGA/PDO nonwoven showed strong disintegration, leading to an increase in the observed mass loss due to the fact that fragments of the material may have been lost during media changes. To visualize this effect, macro images at the different degradation stages were taken (Figure 2). 
a) PLGA/PDO
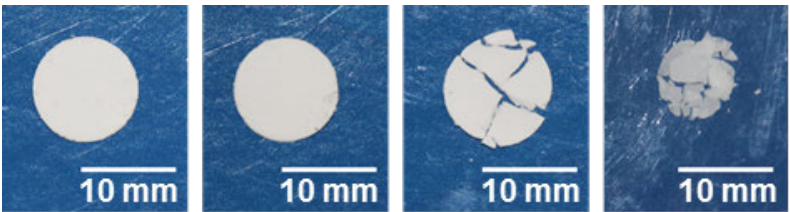

b) PCL/PDO
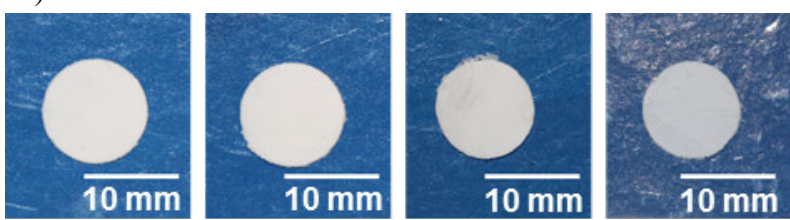

Figure 2: Macro photography of the nonwovens at different degradation time points (from left to right: 7 , 10,14 and 21 days). The scale bar represents $10 \mathrm{~mm}$.

For a more detailed insight on the underlying chemical processes during the degradation, the nonwoven samples were further analysed via GPC to determine the loss in molecular weight. As it can be seen in Figure 3, both composite materials show a similar behavior regarding degradation of the polymer chains in a time range of $0-14$ days. This, however, is in strong contrast to the results of the total mass lost experiment. We assume that this contradiction is based on a more pronounced macroscopic cleavage of the nonwoven fibers for the PLGA/PDO nonwoven compared to the PCL/PDO system. However, this observation has to be addressed more in detail in the on-going work.

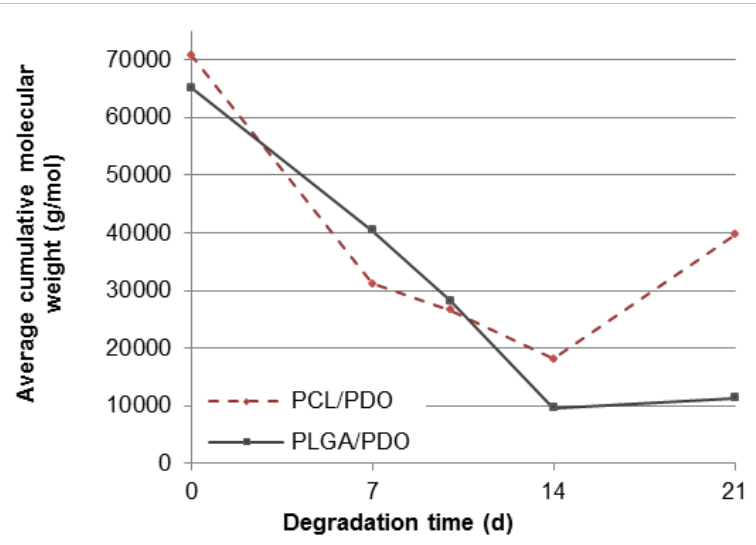

Figure 3: Cumulative molecular weight loss during accelerated degradation of PLGA/PDO (grey) and $\mathrm{PCL} / \mathrm{PDO}$ (red/dashed) composite nonwoven in glycine buffer at $\mathrm{pH}=8.7$ and $50{ }^{\circ} \mathrm{C}(\mathrm{n}=2$ with 3 repetitive measurements each)

Regarding the last data point, the GPC measurements showed an increase in MW for both composite materials at
21 days. This observation can be explained by the composition of the materials. The nonwovens consist of two fiber types from two distinct polymers which exhibit different degradation behaviors, whereas on polymer degrades more slowly compared to the other. This leads to the formation of two overlapping signals in the chromatogram, causing a distortion on the outcome of the MW determination. A representative image of such a chromatogram is shown in Figure 4.

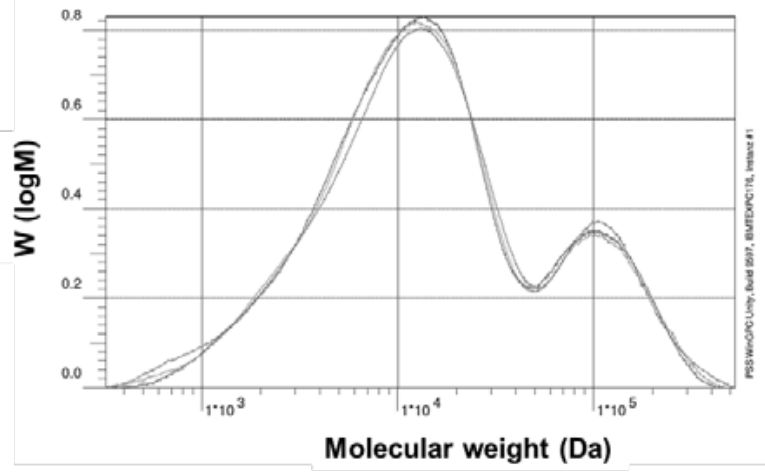

Figure 4: Overlapping gel permeation chromatograms for PCL/PDO composite nonwoven after accelerated degradation for $21 \mathrm{~d}$. The image shows the results for all three repetitive measurements for one sample.

\section{Conclusion}

The combination of two different polymers to form a composite nonwoven via dual co-electrospinning is a promising way to generate matrices for GTR. Our initial experiments showed that the choice of the combined polymers crucially alters the degradation behavior of such nonwovens. Although the molecular mass loss is comparable, PLGA/PDO showed a distinctly higher degree of fragmentation and total mass loss compared to a PCL/PDO composite.

Follow-up work of our group will focus more on these effects, e.g. by SEM imaging of the degrading samples to investigate changes in fiber morphology, which may be responsible for the observed differences in behaviour. Furthermore, cell seeding experiments will be conducted to evaluate the suitability of polyester nonwoven composites for possible in vivo applications.

\section{Acknowledgements}

The skillful work of Annika Volpert, Manfred Strotmeier and Jonathan Ortelt is gratefully acknowledged. The authors 
thank Evonik Industries for kindly providing the Resomer samples.

\section{Author Statement}

Research funding: Research funding: Financial support by the Federal Ministry of Education and Research (BMBF) within RESPONSE "Partnership for Innovation in Implant Technology" is gratefully acknowledged. Conflict of interest: Authors state no conflict of interest. Informed consent: Informed consent is not applicable. Ethical approval: The conducted research is not related to either human or animal use.

\section{References}

[1] Bottino MC, Thomas V, Schmidt G, Vohra YK, Chu TM, Kowolik MJ, Janowski GM. Recent advances in the development of GTR/GBR membranes for periodontal regeneration- a materials perspective. Dent Mater 2012;28:703-721.

[2] Yin GB, Zhang YZ, Wu JL, Wang SD, Shi DB, Dong ZH. Study on Electronspun Poly(lactic acid) Fibroin-Gelatin Composite Nanofibers Scaffold for Tissue Engineering. J Fiber Bioeng Inform 2009;2:182-188.
[3] Zhang E, Zhu C, Yang J, Sun H, Zhang X, Li S, Wang Y, Sun L, Yao F. Electrospun PDLLA/PLGA composite membranes for potential application in guided tissue regeneration. Mater Sci Eng C Mater Biol Appl 2016;58:278-285.

[4] Zhang S, Huang Y, Yang X, Mei F, Ma Q, Chen G, Ryu S, Deng $X$. Gelatin nanofibrous membrane fabricated by electrospinning of aqueous gelatin solution for guided tissue regeneration. J Biomed Mater Res 90A 2009;3:671-679.

[5] Magiera A, Markowski J, Menaszek E, Pilch J, Blazewicz S. PLA-Based Hybrid and Composite Electrospun Fibrous Scaffolds as Potential Materials for Tissue Engineering. J Nanomatter 2017.

[6] Illner S, Arbeiter D, Teske M, Khaimov V, Oschatz S, Senz V, Schmitz KP, Grabow N, Kohse S. Tissue biomimicry using cross-linked electrospun nonwoven fiber composites. Curr Dir Biomed Eng 2019;5:119-122.

[7] Evonik Industries - "RESOMER $®$ BIORESORBABLE POLYMERS FOR MEDICAL DEVICES", [https://healthcare. evonik.com/product/health-care/en/products/biomaterials/res omer/pages/medical-devices.aspx], Access date: 23.03.2020

[8] Martins JA, Lach AA, Morris HL, Carr AJ, Mouthuy PA. Polydioxanone implants: A systematic review on safety and performance in patients. J Biomater Appl. 2020;34(7):902916. 